\title{
Translation, validity and reliability of the British Sign Language (BSL) version of the EQ-5D-5L
}

\author{
Katherine D. Rogers ${ }^{1} \cdot$ Mark Pilling $^{2} \cdot$ Linda Davies $^{3} \cdot$ Rachel Belk $^{1}$. \\ Catherine Nassimi-Green ${ }^{1} \cdot$ Alys Young ${ }^{1}$
}

Accepted: 22 January 2016/Published online: 18 February 2016

(c) The Author(s) 2016. This article is published with open access at Springerlink.com

\begin{abstract}
Purpose To translate the health questionnaire EuroQol EQ-5D-5L into British Sign Language (BSL), to test its reliability with the signing Deaf population of BSL users in the UK and to validate its psychometric properties.

Methods The EQ-5D-5L BSL was developed following the international standard for translation required by EuroQol, with additional agreed features appropriate to a visual language. Data collection used an online platform to view the signed (BSL) version of the tests. The psychometric testing included content validity, assessed by interviewing a small sample of Deaf people. Reliability was tested by internal consistency of the items and testretest, and convergent validity was assessed by determining how well EQ-5D-5L BSL correlates with CORE-10 BSL and CORE-6D BSL.

Results The psychometric properties of the EQ-5D-5L BSL are good, indicating that it can be used to measure health status in the Deaf signing population in the UK. Convergent validity between EQ-5D-5L BSL and CORE-
\end{abstract}

Katherine D. Rogers

katherine.rogers@manchester.ac.uk

1 Social Research with Deaf People (SORD), School of Nursing, Midwifery and Social Work, University of Manchester, Jean McFarlane Building, Oxford Road, Manchester M13 9PL, UK

2 School of Nursing, Midwifery and Social Work, University of Manchester, Jean McFarlane Building, Oxford Road, Manchester M13 9PL, UK

3 Institute of Population Health, University of Manchester, Jean McFarlane Building, Oxford Road, Manchester M13 9PL, UK
10 BSL and CORE-6D BSL is consistent, demonstrating that the BSL version of EQ-5D-5L is a good measure of the health status of an individual. The test-retest reliability of EQ-5D-5L BSL, for each dimension of health, was shown to have Cohen's kappa values of $0.47-0.61$; these were in the range of moderate to good and were therefore acceptable.

Conclusions This is the first time EQ-5D-5L has been translated into a signed language for use with Deaf people and is a significant step forward towards conducting studies of health status and cost-effectiveness in this population.

Keywords EQ-5D-5L - Psychometric properties - Deaf population $\cdot$ British Sign Language $\cdot$ Translation

\section{Background}

Interest in the health status of populations, in particular cultural groups such as the Deaf population, continues to rise. The Deaf population (with a capital 'D') concerns Deaf people who use sign language and identify themselves as part of the Deaf community. Sign languages are not universal, and, in the UK, the language used by Deaf people is British Sign Language (BSL). Previous research on the health of Deaf populations mostly focused on mental well-being [1-3], although there has also been a recent growth in the literature about deficits in the physical health of Deaf people. Studies demonstrate that Deaf people have poorer mental and physical health than the majority population of hearing people and that they experience inequalities in accessing healthcare services [4]. Communication difficulties between healthcare providers and patients, patients' lack of access to health care in their 
preferred language and poor health-related information in signed languages are the main factors $[5,6]$.

The health status of people within the general population can be measured using a standardised assessment tool developed by EuroQol: the EQ-5D (http://www.euroqol. org/eq-5d-products.html) and subsequently the EQ-5D-5L, the latter being considered more robust because it produces fewer ceiling and floor effects [7]. The EQ-5D-5L is a selfreport tool which includes five dimensions of health: Mobility, Self-Care, Usual Activities, Pain/Discomfort and Anxiety/Depression, and a Visual Analogue Scale (VAS) designed to give an overall, self-report summary evaluation of an individual's health status.

Although population norms are available for the EQ-5D for the UK in its English (three level) version [8], the Deaf population in the UK use BSL as their first or preferred language and constitute a separate cultural community $[9$, 10]. Theoretically, English in its written form would seem to present no barriers to access because it is not dependent on hearing, but it is not an appropriate format for a population whose main language is other than English [11]. The socio-economic, educational and cultural experiences of Deaf people are also different from mainstream society $[12,13]$; therefore, the value of scores developed for the general population in the UK is questionable. There is currently no version of the EQ-5D in BSL, or in any other signed language.

The EQ-5D can be used to estimate health benefits in terms of quality-adjusted life years (QALYs) for use in economic evaluations to assess the relative cost-effectiveness of healthcare interventions [14]. The QALY is the measure of health benefit preferred by National Institute for Health and Care Excellence (NICE) for such analyses [14]. Where the population's set of health preferences are not known, then 'one from a nearby or "similar" population' [15] can be used. Deaf populations are not similar to those with hearing loss because the latter group will not use a signed language and are not members of the cultural community denoted by BSL. This means that further investigation is required to identify (or, if necessary, develop) a generic health status measure that is relevant and culturally appropriate for the Deaf population.

The study aims were to: (1) translate the English version of EQ-5D-5L into BSL; (2) validate the EQ-5D-5L BSL on a Deaf population of BSL users in the UK; (3) investigate the psychometric properties of the EQ-5D-5L BSL to establish its reliability. This provides a basis for further research to validate existing norms for the health domains and preference (utility) weights attached to the EQ-5D which were developed for the general population. This work, however, was outside the scope of this project.

\section{Methods}

\section{The translation}

Work on the translation and reliability testing of various standard assessments into BSL (including the CORE-OM, PHQ-9 and GAD-7) has previously been carried out by the authors and specific challenges resulting from translating from a written form into a visual form of a language discussed [13, 16, 17]. The resulting robust translation protocols arising from previous work were applied to the translation of the EQ-5D-5L. Two translation teams were established. The forward translation team consisted of two native Deaf BSL users who are experienced translators, fluent in written English; the back translation team consisted of two registered interpreters (one Deaf and one hearing) who are bilingual in BSL and English. Both teams translated from their second language into their first. The work was overseen by a native BSL user (the first author) who is bilingual in BSL and English. The EuroQol group translation guidelines [18] were adhered to, but adapted to take into account the fact that BSL is a visual (non-written) language. This meant that each stage of the translation procedure was filmed and recorded to allow comparisons between versions. Team discussions concerning discrepancies between forward and back translations were also carried out in BSL and filmed so that points could be referred back to when considering amendments. Team discussions resulted in consensus on the translation of each item to be used in the subsequent draft having considered reasons underlying any differences between the forwardand back-translated versions.

\section{Forward translation}

The two forward translators independently translated the EQ-5D-5L into BSL (first draft). A key problem identified concerned the repetition of the level descriptors; in the English version, these are distinguished by slight changes in the adjective used in each sentence, e.g. 'I have no problems in walking about; I have slight problems in walking about; I have moderate problems in walking about, etc'. In a written language, this format works because someone reading the questionnaire is able to scan between the level descriptors, which are all on the same page, make comparisons and reach a decision; there is simultaneous presentation of available choices. In a visual language, where the 'text' of the questionnaire is presented on screen via an online interface, repeating the level descriptors one after another is a sequential experience for the 'viewer'. To compare the different options would require flicking 
between five different videos, separately presented on screen, which is not an equivalent cognitive task to the written version where there is simultaneous access to the range of responses from which to choose. After discussion with the forward translators and the representatives from the EuroQol group, a change in the format of presentation of the potential responses was permitted. The five-level descriptors are, for each domain, presented by a single signed phrase in the form of: 'the health domain (e.g. mobility difficulties) followed by none; slight; moderate; severe; unable/can't'. The grammar of BSL permits intensity to be marked in increasing degrees, having established the core subject first, through inflecting facial expression, handshape, movement and, in some instances, location of signing [16]. The viewer is able to see all possible choices of response simultaneously (as a reader of written text might) and come to their decision. Those taking the assessment give their response by clicking on one of the available choices represented on screen by corresponding English words (see Fig. 1 for a screen shot), and a BSL reference translation is given at the start and available to be seen again throughout if required.

\section{Back translation}

The two back translators independently translated the BSL version (second draft) back into English, compared the back translations with the original version and produced a report on the back translation process to the project manager. This resulted in the third draft.

\section{Respondent testing}

The third draft was tested using a sample of eight lay Deaf respondents (five men and three women), aged between 33 and 58, with varying educational backgrounds. They included both healthy people and patients, as outlined by the EuroQol group. The 'patients' were those who reported that they were currently experiencing health difficulties in response to general descriptive questions about their health and any current treatment. They were asked to complete the EQ-5D-5L BSL, not having had previous access to or experience of the assessment in English, and then they took part in a structured interview. Additionally, they completed a rating exercise (on a scale of $0-100$ ) to establish their response for each health dimension, which confirmed that the severity descriptors for each dimension were appropriate. Feedback resulted in some additional changes to the translation, including clarification of the acronym EQ-5D, a clearer distinction between pain and/or discomfort and a greater emphasis on 'today' to be conveyed for each dimension as it was signed.

\section{Testing with bilingual Deaf people}

To explore agreement between the English and BSL versions of EQ-5D, 11 bilingual Deaf people completed both versions. Cohen's kappa $(k)$ statistic was used to assess the level of agreement between the English and BSL versions. Although the sample was small, it was found that the level of agreement between the two versions was very high. Statistical agreement (Cohen's kappa) between the BSL
Fig. 1 Example of the onscreen EQ-5D-5L in BSL

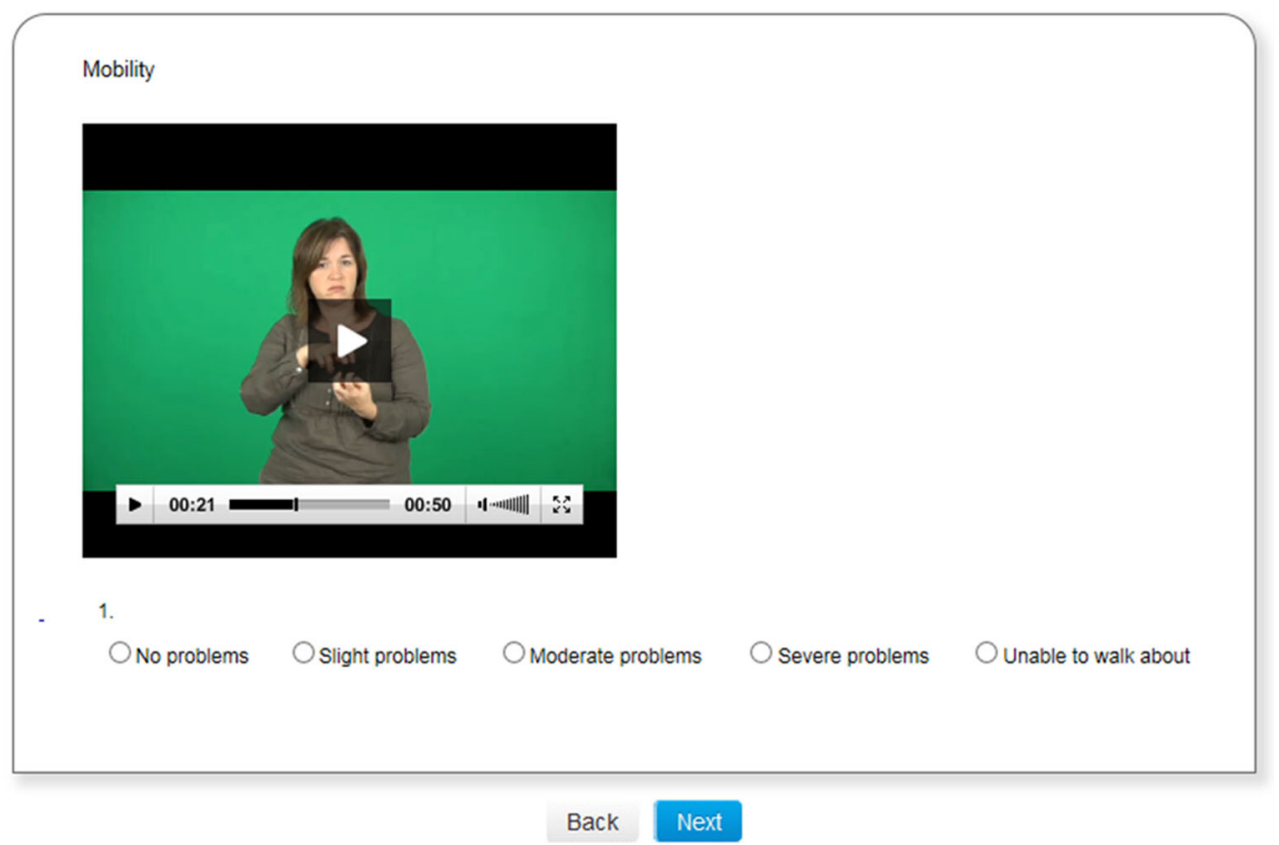


and English versions of each dimension of EQ-5D-5L was high: Mobility, $k=1$ ( $p<0.001)$; Usual Activities, $k=1$ $(p<0.001) ; \quad$ Pain/Discomfort, $k=0.81 \quad(p<0.001)$; Anxiety/Depression, $k=1(p<0.001)$. For Self-Care, all respondents used only one category of the five levels for the English and BSL versions; thus, it was not possible to estimate Cohen's kappa. The analyses above demonstrate that the content of each item in the BSL version of EQ-5D5L was equivalent to the English items in the original version.

\section{Fourth draft}

This took into account comments from the respondent testing and the EuroQol translation review team and was the version then used for reliability testing. Examples of the amendments made include: (i) emphasising more strongly that the question is asking about the severity of problems for today only; (ii) making it clearer that the mobility domain refers to the ability to walk rather than barriers to mobility including communication barriers; and (iii) making the distinction between pain and discomfort clearer.

\section{Investigating the validity and reliability of the BSL version of EQ-5D-5L}

Draft four of EQ-5D-5L BSL was uploaded to an adapted web platform, 'Selectsurvey', which allows videos to be embedded within it (https://selectsurvey.net/). This remote data capture technique is time-consuming and cost-effective and appropriate for a geographically dispersed, small linguistic community such as the Deaf community [13] whilst accommodating the visual modality of the language.

\section{Sample size estimates}

For the test-retest of EQ-5D-5L BSL (at baseline and one week later), an intra-class correlation coefficient (ICC) of at least 0.7 was required to establish reliability. Conventions used were 'poor' for ICC values less than 0.40, 'fair' for values between 0.40 and 0.59 , 'good' for values between 0.60 and 0.74 and 'excellent' for values between 0.75 and 1.0 [19]. Typically, 0.7 is the minimum acceptable for research purposes [20]. A sample size of 51 allows a $95 \%$ confidence interval for an ICC of 0.75 to be estimated to within plus or minus 0.1 . The aim was to recruit 75 people in case of incomplete data. Previous studies demonstrated that this sample size, utilising the same method of recruitment, was entirely feasible [17, 21].

\section{Recruitment}

Participants were recruited using email, Facebook, word of mouth/hands and online message boards read or watched by Deaf people. For the purposes of assessing the reliability and validity of the BSL EQ-5D-5L within a participant sample, it was felt that the benefits of increased sample size from this recruitment approach outweighed the risks of selection bias. Inclusion criteria were: 18 years old or older and a Deaf BSL user. All information and consent materials were available in BSL, with an option for direct contact with a native BSL user for further clarification. Informed consent was obtained from participants online prior to completing the assessments, EQ-5D-5L BSL, CORE-10 BSL, CORE-6D BSL (see below) and this included consent to contact a participant's GP (General Practitioner). If a participant gave an answer other than 'never' to the CORE-10 BSL question about suicidal intent, the research team regarded this as a flag for concern.

\section{Materials and procedure}

Participants completed a short demographic survey, the EQ-5D-5L BSL and the CORE-10 BSL and CORE-6D BSL. Included in the demographic survey were questions relating to:

- Age and gender;

- Parental hearing status (an indicator of whether someone grew up with BSL as native language);

- A self-report of their current difficulties (if any) with their physical and/or mental health.

EQ-5D-5L was presented in a self-report on-screen format in BSL and accessed online. It has five levels of response (no problems, slight problems, moderate problems, severe problems and extreme problems) for each of the five dimensions of health. The standard EQ-VAS was also included. This asked the participants to rate their health on the day from 0 ('the worst health state you can imagine') to 100 (the best health state you can imagine'). The VAS was portrayed as an on-screen thermometer with a button that was moved to choose the placement upon it and then automatically captured the number relating to this position. In addition, there was a box for a participant to write in their choice of number from 0 to 100 . Both approaches were used because the printed English version of EQ-5D-5L asks the participant to mark an $\mathrm{X}$ on a scale and to write the number from the scale into a box.

The CORE-10 [22] and the CORE-6D are self-report instruments designed to be used as screening tools for psychological distress; all items in both derive from the CORE-OM, which has previously been translated into BSL and its reliability demonstrated [21]. Two items in CORE- 
10 BSL and CORE-6D BSL are the same and were not duplicated. Therefore, a total of 14 items were presented on screen in their BSL form as previously established from the CORE-OM BSL. The CORE-10 BSL and the CORE-6D BSL have five levels of response: $(0=$ Never, $1=$ Rarely, 2 = Sometimes, $3=$ Often, $4=$ Mostly/Always). The maximum possible score for the CORE-10 BSL is 40 and the lowest possible is 0 ; the maximum possible score for the CORE-6D BSL is 24 and the minimum 0 .

\section{Analysis}

The frequencies and percentages of responses on all items were calculated. A value of 1 on the EQ-5D-5L index score $=$ 'perfect health' and $0=$ 'as bad as death'. The CORE-10 BSL responses were combined into a single unweighted score. This was calculated as the sum of the item scores divided by the number of questions answered. CORE-6D BSL is not a standalone measure. It was only used to report convergent validity with the EQ-5D-5L BSL.

Published population tariffs for the EQ-5D 3 level [23, 24] and the published crosswalk calculator (http://www. euroqol.org/about-eq-5d/valuation-of-eq-5d/eq-5d-51-valuesets.html) were used to generate a preference weight for each possible combination of the EQ-5D-5L items and levels [25]. The index is calculated by deducting the appropriate weights from 1 , giving a range of 1 or less. Negative values (reflecting health states considered to be worse than death) are possible [24]. The mean utility index score for the UK is 0.856 [24].

The published population norms for the UK were compared with results from the Deaf population sample. However, these are for the 3 level version of the EQ-5D [23], the study sample is relatively small, and the comparisons are not standardised for age and gender; therefore, only percentages with 'no problems' in each health domain are compared.

Cronbach's alpha values were calculated to assess internal reliability of the translated items within EQ-5D-5L BSL, the five attributes of which are treated as different facets of the single construct of health-related well-being. One week after their first completion, participants were asked to complete the EQ-5D-5L BSL again to calculate reliability over time [by calculating the interclass correlation coefficient (ICC) using an absolute two-way mixed estimator]. Values above 0.75 are considered as an 'excellent' agreement between the first and second tests [26].

Weighted kappa scores were used to examine the reliability for the individual items of EQ-5D-5L BSL between the first and second tests: $<0.20$ (poor), $0.21-0.40$ (fair), $0.41-0.60$ (moderate), $0.61-0.80$ (good), 0.81-1.00 (very good) [27].
The CORE-6D BSL and CORE-10 BSL measure aspects of health, as does the EQ-5D-5L; therefore, an overall relationship might be expected and a stronger relationship expected between specific items in each instrument measure. Five questions in CORE-10 BSL and one in the CORE 6D BSL cover the Anxiety/Depression domain; one question in CORE-6D covers Pain/Discomfort domain, and one question in CORE-6D covers the functioning domain of the EQ-5D-5L BSL. Kendall's tau was used to assess the correlation between related items within each tool, which is a more robust estimator than Spearman's rank correlation or Pearson's correlation, especially on smaller sample sizes.

Discussion with the EuroQol representatives indicated they would expect an association between concurrent disability or health problems. Known-groups analysis, using the Mann-Whitney $U$ test, was performed to confirm whether this existed or not. However, the sample size used in this study was not sufficient to assess whether participants' demographic characteristics were statistically associated with the EQ-5D utility index.

\section{Results}

\section{Interviews from the respondent testing stage}

The interviews with eight Deaf people at the respondent testing stage indicated that how Deaf people understand health-related concepts is, in some cases, influenced by their experiences of communication. For example, a few people explained that, to them, 'mobility' encompassed the use of public transport (how easy would it be for a Deaf person to get around?). One respondent stated that he would select 'slight problems' because of the communication barriers rather than considering it from the perspective of physical ability. For others, when considering the Anxiety/Depression domain, they also were considering the linguistic accessibility of mental health services as an influence on their response, not just their internal distress.

\section{Psychometric properties of EQ-5D-5L BSL}

One hundred people participated in the first test of EQ-5D5L BSL (draft four version). Eight did not meet the inclusion criteria and were excluded from the data analysis; they either did not report their hearing status or were 'hard of hearing' (i.e. did not use BSL). Seventy-four of the original 92 Deaf people returned to take part in the retest. Of 100 people who originally completed the first survey, 18 responses to the suicidal intent question contained within 
CORE-10 ('I have made plans to end my life') triggered the study protocol to contact them and alert their primary care physician: 16 of these were included in the analysis; the remaining 2 did not meet the inclusion criteria.

Table 1 presents the demographic characteristics of the sample.
Nearly all had qualifications at GCSE level or above $(95.3 \%)$ and $41.2 \%$ had at least an undergraduate degree or equivalent qualification. On a five-point scale, the majority of participants $(78.3 \%)$ identified with the two highest categories of considering themselves as "culturally Deaf'. A majority also reported that they are 'often' or
Table 1 Demographic profile of participants

\begin{tabular}{|c|c|c|}
\hline & First test $n=92$ & Test-retest $n=74$ \\
\hline \multicolumn{3}{|l|}{ Gender } \\
\hline Female & $64(69.6 \%)$ & $52(70.3 \%)$ \\
\hline Male & $26(28.3 \%)$ & $21(28.4 \%)$ \\
\hline Missing data & $2(2.2 \%)$ & $1(1.4 \%)$ \\
\hline \multicolumn{3}{|l|}{ Age } \\
\hline $18-24$ & $4(4.3 \%)$ & $4(5.4 \%)$ \\
\hline $25-34$ & $14(15.2 \%)$ & $10(13.5 \%)$ \\
\hline $35-44$ & $17(18.5 \%)$ & $14(18.9 \%)$ \\
\hline $45-54$ & $26(28.3 \%)$ & $22(29.7 \%)$ \\
\hline $55-64$ & $14(15.2 \%)$ & $11(14.9 \%)$ \\
\hline $65+$ & $3(3.3 \%)$ & $2(2.7 \%)$ \\
\hline Missing data & $14(15.2 \%)$ & $11(14.9 \%)$ \\
\hline \multicolumn{3}{|l|}{ Ethnicity } \\
\hline Asian or Asian British: Indian & $4(4.3 \%)$ & $2(2.7 \%)$ \\
\hline Asian or Asian British: Pakistani & $3(3.3 \%)$ & $1(1.4 \%)$ \\
\hline Black or Black British: other Black background & $1(1.1 \%)$ & $1(1.4 \%)$ \\
\hline Mixed: any other mixed background & $1(1.1 \%)$ & $1(1.4 \%)$ \\
\hline Other ethnic group & $3(3.3 \%)$ & $1(1.4 \%)$ \\
\hline White: any other white background & $4(4.3 \%)$ & $4(5.4 \%)$ \\
\hline White: British & $71(77.2 \%)$ & $61(82.4 \%)$ \\
\hline White: Irish & $2(2.2 \%)$ & $1(1.4 \%)$ \\
\hline Missing data & $3(3.3 \%)$ & $2(2.7 \%)$ \\
\hline \multicolumn{3}{|l|}{ Parents Deaf? } \\
\hline Yes & $24(26.1 \%)$ & $21(28.4 \%)$ \\
\hline No & $68(73.9 \%)$ & $53(71.6 \%)$ \\
\hline \multicolumn{3}{|l|}{ Age first used BSL } \\
\hline From birth & $22(23.9 \%)$ & $18(24.3 \%)$ \\
\hline $1-3$ years old & $20(21.7 \%)$ & $15(20.3 \%)$ \\
\hline 4-7 years old & $15(16.3 \%)$ & $13(17.6 \%)$ \\
\hline $8-11$ years old & $6(6.5 \%)$ & $6(8.1 \%)$ \\
\hline $12-16$ years old & $7(7.6 \%)$ & $7(9.5 \%)$ \\
\hline $17-24$ years old & $13(14.1 \%)$ & $7(9.5 \%)$ \\
\hline $25+$ years old & $9(9.8 \%)$ & $8(10.8 \%)$ \\
\hline \multicolumn{3}{|l|}{ Currently in employment } \\
\hline Yes & $63(68.5 \%)$ & $52(70.3 \%)$ \\
\hline No & $28(30.4 \%)$ & $21(28.4 \%)$ \\
\hline Missing data & $1(1.1 \%)$ & $1(1.4 \%)$ \\
\hline \multicolumn{3}{|l|}{ Health difficulties } \\
\hline Yes & $26(28.3 \%)$ & $21(28.4 \%)$ \\
\hline No & $58(63 \%)$ & $47(63.5 \%)$ \\
\hline I don't know & $8(8.7 \%)$ & $6(8.1 \%)$ \\
\hline
\end{tabular}


Table 2 Frequencies and percentages of responses for items within EQ-5D-5L BSL, CORE-10 BSL

\begin{tabular}{|c|c|c|c|c|c|c|}
\hline \multicolumn{7}{|c|}{ Based on $n=92, N(\%)$} \\
\hline \multicolumn{2}{|c|}{ EQ-5D-5L health states } & None & Slight & Moderate & Severe & Extreme \\
\hline \multicolumn{2}{|c|}{ EQ-5D-5L Mobility } & $63(68.5)$ & $18(19.6)$ & $6(6.5)$ & $5(5.4)$ & $0(0)$ \\
\hline \multicolumn{2}{|c|}{ EQ-5D-5L Self-Care } & $80(86)$ & $9(9.7)$ & $2(2.2)$ & $2(2.2)$ & $0(0)$ \\
\hline \multicolumn{2}{|c|}{ EQ-5D-5L Usual Activities } & $57(61.3)$ & $26(28)$ & $6(6.5)$ & $3(3.2)$ & $1(1.1)$ \\
\hline \multicolumn{2}{|c|}{ EQ-5D-5L Pain/Discomfort } & $45(48.4)$ & $31(33.3)$ & $7(7.5)$ & $6(6.5)$ & $4(4.3)$ \\
\hline \multicolumn{2}{|c|}{ EQ-5D-5L Anxiety/Depression } & $43(46.2)$ & $31(33.3)$ & $15(16.1)$ & $4(4.3)$ & $0(0)$ \\
\hline $\begin{array}{l}\text { CORE-10 } \\
\text { states }\end{array}$ & Never & Rarely & Sometimes & Often & \multicolumn{2}{|c|}{ Mostly/always } \\
\hline CORE-10 Q1 & $23(26.4)$ & $26(29.9)$ & $28(32.2)$ & $8(9.2)$ & \multicolumn{2}{|c|}{$2(2.3)$} \\
\hline CORE-10 Q2 & $13(14.9)$ & 16 (18.4) & $25(28.7)$ & $16(18.4)$ & \multicolumn{2}{|c|}{$17(19.5)$} \\
\hline CORE-10 Q3 & $11(12.8)$ & $23(26.7)$ & $27(31.4)$ & $12(14)$ & \multicolumn{2}{|c|}{$13(15.1)$} \\
\hline CORE-10 Q4 & $36(40.9)$ & $20(22.7)$ & $27(30.7)$ & $5(5.7)$ & \multicolumn{2}{|c|}{$0(0)$} \\
\hline CORE-10 Q5 & $45(51.1)$ & $24(27.3)$ & $14(15.9)$ & $5(5.7)$ & \multicolumn{2}{|c|}{$0(0)$} \\
\hline CORE-10 Q6 & $68(79.1)$ & $13(15.1)$ & $4(4.7)$ & $1(1.2)$ & \multicolumn{2}{|c|}{$0(0)$} \\
\hline CORE-10 Q7 & $23(26.7)$ & 27 (31.4) & 24 (27.9) & $12(14)$ & \multicolumn{2}{|c|}{$0(0)$} \\
\hline CORE-10 Q8 & $20(23)$ & $30(34.5)$ & $31(35.6)$ & $6(6.9)$ & \multicolumn{2}{|c|}{$0(0)$} \\
\hline CORE-10 Q9 & 17 (19.5) & $28(32.2)$ & $34(39.1)$ & $8(9.2)$ & \multicolumn{2}{|c|}{$0(0)$} \\
\hline CORE-10 Q10 & $49(57)$ & $18(20.9)$ & $16(18.6)$ & $2(2.3)$ & \multicolumn{2}{|c|}{$1(1.2)$} \\
\hline
\end{tabular}

'very much' involved in the Deaf community (85.9\%) and have 'a sense of community belonging' (75\%). Nine per cent reported that they did not know whether they had any health difficulties or not. Table 2 presents the frequencies and percentages of responses on all items at the first test.

The distribution of the EQ-5D-5L BSL utility index was skewed and the median value was used to represent the average, which is 0.84 with $95 \%$ CI [0.72-0.82 bias-corrected accelerated bootstrap $(\mathrm{BCa})] \quad($ mean $=0.78$, $\mathrm{SD}=0.24, \quad \mathrm{IQR}=0.72-1.00)$. The mean score for CORE-10 BSL is $11.74(\mathrm{SD}=5.31)$ and was not skewed (for reference, the median $=11.50$ with $95 \% \quad \mathrm{CI}$ (10.64-13.12) BCa, IQR = 8.0-16.0).

The mean utility index score for EQ-5D-5L BSL in this study was 0.78 . The percentage of the study sample with 'no problems' in each health domain was less than the UK population published norms (Fig. 2).

The Cronbach's alpha values for EQ-5D-5L BSL showed that the internal reliability is ' $g$ ood' $[\alpha=0.86$ with $95 \%$ CI (0.80-0.90), $n=89$, for initial test; and $\alpha=0.82$ with $95 \%$ CI (0.74-0.88), $n=72$, for test-retest] and for CORE-10 BSL 'acceptable' $[\alpha=0.72$ with $95 \%$ CI (0.62-0.80), $n=78$, for initial test; and $\alpha=0.75$ with $95 \%$ CI (0.65-0.83), $n=70$, for test-retest] [28]. These values showed that there is good internal agreement between the items for each measure, but not so much agreement to suggest one or more items were redundant.

Participants were asked to repeat the measures one week later although some took longer. A sensitivity analysis on

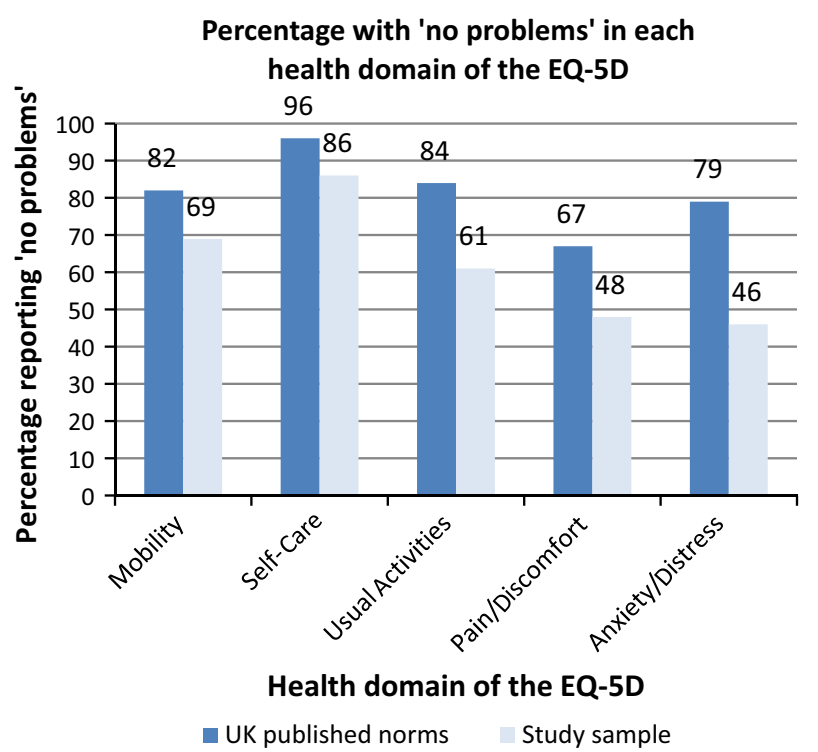

Fig. 2 Bar chart showing the percentage with 'no problems' in each health domain of the EQ-5D

three subsets of the data showed that the values are consistent regardless of the time it took to retest: all data ( $n=74)$ ICC $=0.87$; two weeks or less to retest $(n=63)$ $\mathrm{ICC}=0.87$; seven days $=/-$ three days to retest $(n=50)$ ICC $=0.86$. The ICC for VAS and the typed score between two time points were also considered as excellent and good, respectively [ICC $=0.82 \quad(n=72)$ and ICC $=0.64(n=60)$, respectively $]$. 
Table 3 Weighted kappa values by question between first and second tests of EQ-5D-5L BSL

\begin{tabular}{ll}
\hline & Weighted kappa $[95 \% \mathrm{CI}]$ \\
\hline EQ-5D-5L BSL Mobility & $0.61(n=73)[0.45,0.77]$ \\
EQ-5D-5L BSL Self-Care & $0.47(n=73)[0.08,0.86]$ \\
EQ-5D-5L BSL Usual Activities & $0.54(n=73)[0.38,0.69]$ \\
EQ-5D-5L BSL Pain/Discomfort & $0.61(n=73)[0.48,0.74]$ \\
EQ-5D-5L BSL Anxiety/ & $0.48(n=72)[0.32,0.64]$ \\
$\quad$ Depression & \\
\hline
\end{tabular}

The reliability for the individual items of EQ-5D-5L BSL between the first and second tests was examined using weighted kappa (see Table 3). The agreement was generally 'moderate' to 'good' [27].

The convergent validity for EQ-5D-5L BSL was assessed by checking how well it correlated with CORE-10 BSL and CORE-6D BSL at the first test $(n=92)$. The EQ-5D5L BSL has a positive Pearson's correlation with CORE-10 BSL and CORE-6D BSL $(r=-0.432, n=78, p<0.001$ and $r=-0.449, n=82, p<0.001$, respectively). Four of the five items from the CORE-10 BSL and two of the three items from the CORE-6D BSL demonstrated mediumstrong correlation $(\geq 0.3)[29]$ in the right direction with the EQ-5D-5L BSL Anxiety/Depression domain. One item from the CORE-10 BSL had a strong correlation $(\geq 0.5)$ with EQ-5D-5L BSL Anxiety/Depression, and one item from the CORE-6D BSL demonstrated a strong correlation in the right direction with the Pain/Discomfort domain of the EQ-5D-5L BSL, but no significant correlation was found between one item from the CORE-6D BSL and the Usual Activities domain of the EQ-5D-5L BSL (Table 4).

The expected association between concurrent disability or health problems (yes/no) and utility weights estimated from the EQ-5D-5L BSL was confirmed (Mann-Whitney
$\mathrm{U}, p<0.001$ ), with better (higher) values on the EQ-5D5L BSL being associated with no problems.

\section{Discussion}

The results demonstrate the psychometric properties of the EQ-5D-5L BSL are good, indicating that it can be used to measure health status and QALYs in the Deaf signing population in the UK. Convergent validity between EQ5D-5L BSL and CORE-10 BSL and CORE-6D is consistent, demonstrating that the BSL version of EQ-5D-5L is a good measure of the health status of an individual. The test-retest reliability of EQ-5D-5L, for each dimension of health, was shown to have Cohen's kappa values of $0.47-0.61$; these were in the range of moderate to good and therefore acceptable.

Cohen's kappa values in EQ-5D-5L BSL for Self-Care, Usual Activities and Anxiety/Depression were moderate, whereas for Mobility and Pain/Discomfort, they were good. The reasons for the moderate values for Self-Care and Usual Activities are not known. In relation to Anxiety/ Depression, one possible explanation for the moderate agreement between the two tests is any emotional changes during the period of retest.

Nearly $9 \%$ of Deaf participants in this study stated they did not know if they had health difficulties, possibly indicating a lack of understanding of what is considered 'being healthy'. This may result from poor access to health-related information generally as so little is available in BSL [6] or any signed language $[4,5]$. The limited interview data from the respondent testing stage indicates that Deaf people's responses to the health domains explored in the EQ-5D-5L may also be mediated by their experiences of communication barriers, with respect to both services and everyday life. Severity ratings of difficulties with mobility or anxiety may be influenced by experiences of whether services are accessible in BSL rather than only the severity of

Table 4 Convergent validity between items from the CORE-10 BSL and the CORE-6D BSL

\begin{tabular}{llrr}
\hline CORE question & EQ-5D-5L domain & Kendall's tau & Fisher's exact test \\
\hline CORE-10 Q1 (tense, anxious or nervous) & Anxiety/Depression & 0.50 & $p<0.001$ \\
CORE-10 Q5 (panic or terror) & Anxiety/Depression & 0.379 & $p=0.001$ \\
CORE-10 Q6 (end life) & Anxiety/Depression & 0.295 & $p=0.002$ \\
CORE-10 Q8 (despairing or hopeless) & Anxiety/Depression & 0.410 & $p<0.001$ \\
CORE-10 Q9 (unhappy) & Anxiety/Depression & 0.319 & $p<0.042$ \\
CORE-6D (I have felt terribly alone and isolated) & Anxiety/Depression & 0.393 & $p<0.001$ \\
CORE-6D (I have been troubled by aches, pains or other physical problems) & Pain/Discomfort & 0.58 & $p<0.001$ \\
CORE-6D (I have been able to do most things I needed to) & Usual Activities & -0.11 & $p=0.366$ \\
\hline
\end{tabular}


symptoms. Further study is required to explore Deaf people's conceptualisation of 'health' and whether experiences of communication barriers mediate personal ratings of healthiness.

Cautious comparisons between study sample results and published EQ-5D UK population norms show a far lower percentage of Deaf people in this sample reported 'no problems' in the health domains of the EQ-5D in comparison with the general UK population. This is consistent with a recent study of Deaf health in the UK [6]. Further research is required to facilitate comparison of EQ-5D health states and utility values for the Deaf population in relation to the general population.

\section{Limitations}

Participants may not be representative of the Deaf population, and collection online restricts the sample.

\section{Conclusion}

This is the first signed version of the EQ-5D-5L instrument. It is a significant step forward in the study of cost-effectiveness and health status of Deaf people.

Acknowledgments This study is funded by the National Institute for Health Research's Health Services and Delivery Research Programme (Grant Number: 12/136/79). This report/article presents independent research commissioned by the National Institute for Health Research (NIHR). The views expressed in this publication are those of the authors and not necessarily those of the NHS, the NIHR or the Department of Health. The authors wish to express thanks to translation team: Rosemary Oram, Nicholas Padden, Jen Dodds and Jim Cromwell, for their work on translating EQ-5D-5L into BSL.

Funding This study was funded by the National Institute for Health Research (NIHR) Health Services Research and Delivery Programme, Grant Award Number: 12/136/79.

\section{Compliance with ethical standards}

Conflict of interest The authors declare that they have no conflicts of interest.

Ethical approval All procedures performed in studies involving human participants were in accordance with the ethical standards of the institutional and/or national research committee and with the 1964 Helsinki Declaration and its later amendments or comparable ethical standards. This article does not contain any studies with animals performed by any of the authors.

Open Access This article is distributed under the terms of the Creative Commons Attribution 4.0 International License (http://crea tivecommons.org/licenses/by/4.0/), which permits unrestricted use, distribution, and reproduction in any medium, provided you give appropriate credit to the original author(s) and the source, provide a link to the Creative Commons license, and indicate if changes were made.

\section{References}

1. Hindley, P., Hill, P. D., McGuigan, S., \& Kitson, N. (1994). Psychiatric disorder in deaf and hearing impaired children and young people: A prevalence study. Journal of Child Psychology and Psychiatry, 35(5), 917-934. doi:10.1111/j.1469-7610.1994. tb02302.x.

2. Kvam, M. H., Loeb, M., \& Tambs, K. (2007). Mental health in Deaf adults: Symptoms of anxiety and depression among hearing and Deaf individuals. Journal of Deaf Studies and Deaf Education, 12(1), 1-7. doi:10.1093/deafed/en1015.

3. Rogers, K. D., \& Young, A. (In press). Is there an association between deaf children's mental health difficulties and their adult well-being? The state of the evidence. In: L. Leeson and M. Vermeerbergen (Eds.), Working with the Deaf Community: Education, Mental Health \& Interpreting. Dublin, Ireland: Intersources Group (Ireland) Limited.

4. Kuenburg, A., Fellinger, P., \& Fellinger, J. (2015). Health care access among deaf people. Journal of Deaf Studies and Deaf Education, advance online publication. doi: 10.1093/deafed/ env042. http://jdsde.oxfordjournals.org/content/early/2015/09/23/ deafed.env042.full.pdf + html. Accessed November 12, 2015.

5. Barnett, S., Klein, J. D., Pollard, R. Q, Jr, Samar, V., Schlehofer, D., Starr, M., et al. (2011). Community participatory research with deaf sign language users to identify health inequities. American Journal of Public Health, 101(12), 2235-2238. doi:10. 2105/AJPH.2011.300247.

6. SignHealth (2014) Sick of it report. England: SignHealth. Retrieved August 21, 2015, from http://www.signhealth.org.uk/ sick-of-it-report-professionals/. Accessed November 12, 2015.

7. Herdman, M., Gudex, C., Lloyd, A., Janssen, M. F., Kind, P., Parkin, D., et al. (2011). Development and preliminary testing of the new five-level version of EQ-5D (EQ-5D-5L). Quality of Life Research, 10, 1727-1736. doi:10.1007/s11136-011-9903-x.

8. Kind, P., Hardman, G., \& Macran, S. (1999). UK population norms for $E Q-5 D$. York: Centre for Health Economics, University of York.

9. Ladd, P. (2003). Understanding Deaf culture: in search of Deafhood. Clevedon: Multilingual Matters.

10. Sutton-Spence, R., \& Woll, B. (1999). The linguistics of British Sign Language: An introduction. Cambridge: Cambridge University Press.

11. Young, A., \& Hunt, R. (2011). NSSCR methods review: Research with d/Deaf people. London: National School of Social Care Research. http://www2.1se.ac.uk/LSEHealthAndSocialCare/pdf/ SSCR\%20Methods\%20Review_9_web.pdf. Accessed November $12,2015$.

12. Leigh, I. (2009). A lens on deaf identities. New York: Oxford University Press.

13. Rogers, K. D. (2013). Deaf people and mental well-being: Exploring and measuring mental well-being in British Sign Language (Doctoral thesis, University of Manchester, Manchester, UK). https://www.escholar.manchester.ac.uk/uk-ac-manscw:209223. Accessed November 12, 2015.

14. National Institute for Clinical Excellence. (2013). Guide to the methods of technology appraisal. https://www.nice.org.uk/article/ pmg9/chapter/Foreword. Accessed November 12, 2015.

15. Bailey, H., \& Kind, P. (2010). Preliminary findings of an investigation into the relationship between national culture and EQ-5D value sets. Quality of Life Research, 19, 1145-1154. doi:10.1007/ s11136-010-9678-5.

16. Rogers, K. D., Young, A., Lovell, K., \& Evans, C. (2013). The challenges of translating the Clinical Outcomes in Routine Evaluation-Outcome Measure (CORE-OM) into British Sign Language. Journal of Deaf Studies and Deaf Education, 18(3), 287-298. doi:10.1093/deafed/ent002. 
17. Rogers, K. D., Young, A., Lovell, K., Campbell, M., Scott, P. R., \& Kendal, S. (2013). The British Sign Language Versions of the Patient Health Questionnaire, the Generalized Anxiety Disorder 7-Item Scale, and the Work and Social Adjustment Scale. Journal of Deaf Studies and Deaf Education, 18(1), 110-122. doi:10. 1093/deafed/ens040.

18. The EuroQol Group. (2013). Translation guidelines for the EQ$5 D$ developed by the EuroQol Group. EuroQol Group.

19. Cicchetti, D. V. (1994). Guidelines, criteria, and rules of thumb for evaluating normed and standardized assessment instruments in psychology. Psychological Assessment, 6(4), 284-290. doi:10. 1037/1040-3590.6.4.284.

20. Nunnally, J. C. (1978). Psychometric theory (2nd ed.). New York: McGraw-Hill.

21. Rogers, K., Evans, C., Campbell, M., Young, A., \& Lovell, K. (2013). The reliability of British Sign Language and English versions of the Clinical Outcomes in Routine Evaluation-Outcome Measure with d/Deaf populations in the UK: an initial study. Health and Social Care in the Community, 22(3), 278-289. doi:10.1111/hsc.12078.

22. Barkham, M., Bewick, B., Mullin, T., Gilbody, S., Connell, J., Cahill, J., et al. (2013). The CORE-10: a short measure of psychological distress for routine use in the psychological therapies.
Counselling and Psychotherapy Research: Linking research with practice, 13(1), 3-13. doi:10.1080/14733145.2012.729069.

23. van Hout, B., Janssen, M. F., Feng, Y.-S., Kohlmann, T., Busschbach, J., Golicki, D., et al. (2012). Interim scoring for the EQ5D-5L: mapping the EQ-5D-5L to EQ-5D-3L value sets. Value in Health, 15(5), 708-715. doi:10.1016/j.jval.2012.02.008.

24. van Reenan, M., \& Janssen, B. (2015). Eq-5D-5L user guide, version 2.1. http://www.euroqol.org/fileadmin/user_upload/Doc umenten/PDF/Folders_Flyers/EQ-5D-5L_UserGuide_2015.pdf. Accessed November 12, 2015.

25. Szende, A., Janssen, B., \& Cabases, J. (Eds.). (2014). Self-reported population health: An international perspective based on $E Q-5 D$. Netherlands: Springer.

26. McDowell, I. (2006). Measuring health: A guide to rating scales and questionnaire (3rd ed.). New York, NY: Oxford University Press.

27. Altman, D. G. (1991). Practical statistics for medical research. London, UK: Chapman and Hall.

28. George, D., \& Mallery, P. (2003). SPSS for windows step by steps: A simple guide and reference. Boston, MA: Allyn and Bacon.

29. Cohen, J. (1988). Statistical power analysis for the behavioral sciences (2nd ed.). New Jersey: Lawrence Erlbaum. 